\title{
Intelligent Technologies for Real-time Biomedical Engineering Applications
}

\author{
Hung Tan Nguyen \\ Electrical Engineering \\ University of Technology, Sydney \\ Sydney, 2007, New South Wales, Australia \\ E-mail: Hung.Nguyen@uts.edu.au
}

\begin{abstract}
Intelligent technolog ies are essential for ma ny biomedical engineering applications in order to cope with a wide vari ety of pat ient conditions or user disabilit $\mathrm{y}$. The development of advanced optimisation training algorithms such as adaptive optimal Bayesian neural networks is particularly useful when only limited train ing data are ava ilable. Two s pecific biomedical engineering applications will be presented. The first application concerns the development of a non-invasive monitor for real-time detection of hypoglycaemic episodes in Type 1 diabetes mellitus patients (T1DM). The second application relates to the deve lopment of real -time hands-free wheelchair control systems using head $\mathrm{m}$ ovement to provide $\mathrm{m}$ obility independence for severely disabled people.
\end{abstract}

Keywords: biomedical engineering; artificial intelligence; hypoglycaemia detection; hands-free wheelchair control.

Reference to this paper should be made as follows: Nguyen, H.T. (2008) 'Intelligent Technologies for Real-time Biomedical Engineering Applications', Int. J. Automation and Control, Vol. x, Nos. x, pp. x.

Biographical notes: Hung Tan Nguyen is a Professor of Electrical Engineering at the University of Technology, Sydney. He is Associate Dean , Research and Development, in the Faculty of Engineering and Director of the Centre for Health Technologies at UTS. He received his BE degree with First Class Honours and University Medal in 1976 and PhD degree in 1980 fro $\mathrm{m}$ the University of Newcastle in Australia. He has been involved with research in the areas of biomedical engineering, advanced control and artificial intelligence for more than 20 years. He has developed several biomedical devices and systems for diabetes, disability, cardiovascular diseases and breast cancer that are leading to improved quality of life and which h ave significant benefits for health care and social economics. 
Hung Tan Nguyen

\section{Introduction}

Intelligent technologies are essential for man y biomedical engineering applications in order to deal with various patient conditions or user disability. The development of adaptive optimal Bayesian neural networks is particularly useful when only limited training data are available. Two s pecific biomedical engineering applications are presented below.

The first application concerns the development of a non-invasive monitor for real-time detection of hyp oglycaemia episodes in Type 1 diabetes mellitus patients (T1DM). Diabetes Control and Com plications Trial (DCCT) Research Group in 1993 [DCCT, 1993] showed that intensive insulin therapy for a m ean of 6 year $\mathrm{s}$ as opposed to conventional therapy significantly lowered the risk for retinopathy by $47 \%$, nephropathy by $54 \%$ and for neuropathy by $60 \%$ [DCCT, 1993; Yale, 2004].

On the other hand, patients assigned to intensive therapy experienced a threefold increase incidence of se vere hypoglycemic episodes over those receiving conventional therapy [DCCT, 1993; DCCT 1995]. Therefore, hypoglycemia proved to be a 1 imiting factor in achieving improved diabetes control.

Symptoms of hypoglycemia arise from the activation of the autonomous central nervous systems (autonomic symptoms) and from reduced cerebral glucose consumption (neuroglycopenic symptoms), some of the latter b eing potentially life threatening. Autonomic symptoms (e.g., tachycardia, palpitations, shakiness, sweating) are activated before neuroglycopenic symptoms (e.g., reduced concentration, blurred vision, dizziness). Autonomic symptoms may provide the initial indication of the presence of hypoglycemia and allow the patient to recognise and correct the ensuing episode [Clarke, 1995].

Nocturnal hypoglycemia is particularly dangerous because sleep reduc es and $\mathrm{m}$ ay obscure autonomic counter-regulatory responses, so that an initially mild episode may become severe. The risk of severe hypoglycemia is high at night, with at least $50 \%$ of all severe episodes occurring during that time [DCCT, 1991].

The second application relates to the development of real-time hands-free wheelchair control systems using head movement to provide mobility independence for severely disabled people.

Conventional electrical wheelchairs are no $\mathrm{t}$ always su fficient to compensate for mobility disabilities. Serious spasticity (cerebral palsy), excessive weak residual physical capacities (tetraplegia), or s ome cognitive impairments (head trauma) would exclude or limit their use.

To overcome the problems associated with joystick control, several interfaces have been designed to replace the joystick. Although it is possible to use voice control [Mazo et al., 1995; Simpson and Levine, 2002; Ha et al., 2 005] or ultrasonic non-contact head controller with a small vocabulary voice recognition system [Coyle, 1995], two oftenused hands-free interfaces are chin sticks a nd sip-and-puff systems. However, the above 
interfaces have many limitations concerning posture, ope rational calibration and reliability when operated by severely disabled users.

The hands-free technologies we have focused over in the past 10 years are based on head movement and thought pattern control. In 1998, we developed an alternative telemetric head movement device for the control of p owered wheelchairs using a tilt sensor and wireless technology [Joseph and $\mathrm{N}$ guyen, 1998]. Since then, we hav e developed several implementations using an embedded Linux implementation [Nguyen et al., 2004], wireless head movement wheelchair control systems using a personal digital assistant [Craig and Nguyen, 2005] or optimised Bayesian neural networks [Nguyen et al., 2006b; Nguyen et al., 2007] and a real-time thought pattern control systems in 2006 and 2007 [Craig and Nguyen, 2006; Craig and Nguyen 2007].

In this paper, for the first biomedical application the development of a $\mathrm{n}$ optimal Bayesian neural network algorithm is o utlined for the detection of hypoglycemia episodes in T1DM children using physiological parameters. For the second biomedical application, the development of optimal Bayesian neural networks for the classification of head direction commands and thought patterns is described.

\section{Methods}

\subsection{Non-invasive hypoglycaemia monitor}

Current technologies used in the diabetes diagnostic testing and self-monitoring market have already been improved to the extent that any additional improvements would be minimal. The next technological advancement in this market is expected to occ ur using non-invasive glucose monitors.

There is a limited number of non-invasive blood glucose monitoring systems currently available but each has specific drawbacks in terms of functioning, cost, reliability and obtrusiveness.

GlucoWatch G2 Biographer from Cygnus Inc is designed to measure glucose levels up to 3 times per hour for 12 hours. The AutoSensor (the disposable component) which is attached to the back of the GlucoWatch monitor and adheres to the skin will provide 12 hours of measurement. The product uses reverse iontophoresis to extract and measure glucose levels non-invasively using interstitial fluid. It has to be calibrated before each measurement period and requires at wo-hour warm-up period. It requires costly disposable components, the gel pads must be replaced after each use, sweating may cause skipped readings, and the measurement has a time delay of about 10-15 minutes. As a result of these limitations this device is no longer available.

We have developed a c ontinuous non-invasive hypoglycemia monitor which uses physiological responses [Nguyen et al., 2006a]. During hypoglycemia, the most profound physiological changes are c aused by activation of the sympathetic nervous system. Among the strongest responses are sweating and increased cardiac output [Gale et al., 
Hung Tan Nguyen

1983; Heller, 1991; Harris et al., 1996]. Experimental hypoglycemia has been shown to prolong QT intervals and dispersion in both non-diabetic subjects and in those with Type 1 and Type 2 diabetes [Marques et al., 1997].

HypoMon ${ }^{\circledR}$ from AIMedics Pty Ltd is a no n-invasive monitor that measures physiological parameters continuously to provide detection of hypoglycemic episodes in Type $1 \mathrm{~d}$ iabetes mellitus patients (T1DM). The system consists of a battery-powered chest belt worn that houses a set of four skin-surface bio-sensor electrodes for the measurement of physiological parameters and a hand-held receiver computer. An alarm system is available for warning various stages of hypoglycemia.

\subsection{Hands-free control of power wheelchairs}

The wheelchair platform is based on a c ommercial powered wheelchair (RollerChair M1). The head movement of a user's head is detected by analysing data from a dual-axis accelerometer installed in a cap worn by the user. Head movement data were collected with a sampling period of $100 \mathrm{~ms}$. Four head direction movements are classified in realtime, corresponding to commands for forward, backward, left and right.

The start of a head movement to control the travel direction of the wheelchair is determined to be the point where the deviation from the neutral position reached $25 \%$ of the maximum value on the relevant axis. The computer interface module consists of the feedback to the user which includes real-time graphical displays of the accelerometer data to allow the user to track the deviation of their head from the neutral position.

\section{Bayesian Neural Networks}

Bayesian neural networks were firstly introduced by MacKay as a practical and powerful means to improve the generalisation of neural networks [MacKay 1992a; MacKay 1992b; Thodberg 1996; Penny and Roberts, 1999]. Compared to a stan dard neural network, the evidence for e ach architecture can be e stimated using the Bayesian framework and no separate validation set is required.

\subsection{Multi-layer neural networks}

Multi-layer neural networks are widely used in engineering applications. With one hidden layer network as shown in Fig. 1, the value of the $k$ th output is computed as follows:

$$
z_{k}(x, w)=f_{0}\left(b_{k}+\sum_{j=1}^{M} w_{k j} f_{0}\left(\bar{b}_{j}+\sum_{i=1}^{d} \bar{w}_{j i} x_{i}\right)\right)
$$


Figure 1 A multi-layer neural network

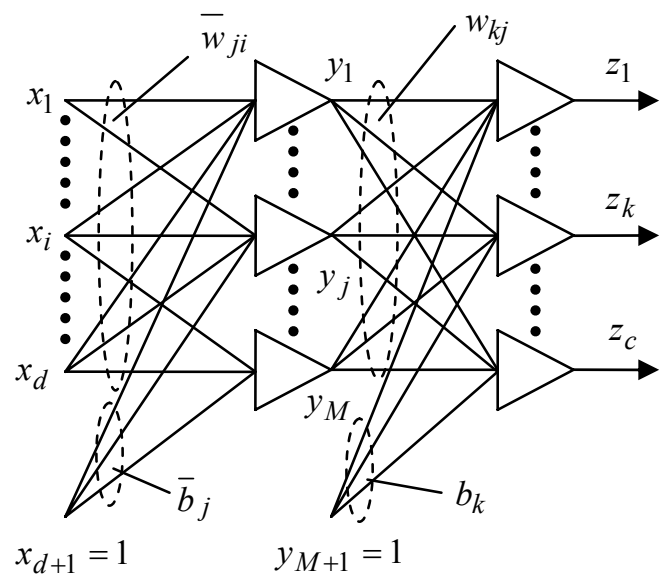

Here, $\bar{w}_{j i}$ is the weight on the connection from input unit $i$ to hidden unit $j$ and $w_{k j}$ is the weight on the connection from hidden unit $j$ to output unit $k . \bar{b}_{j}, b_{k}$ are the biases of the hidden and output units and $f_{0}$ is an activation function.

\subsection{Regularisation}

In neural networks, appropriate regularisation can be used to prevent any weights becoming too large because large weights may give poor generalisation. Therefore, a weight decay term is added to the data e rror function $E_{D}$ to penalise large weights. Specifically, for classification problems, we have:

$$
S(w)=E_{D}+\sum_{g=1}^{G} \xi_{g} E_{W_{g}}
$$

where $S(w)$ is the total error function, $\xi_{g}$ is a non-negative parameter for the distribution of other parameters (weights and biases) and known as a hyperparameter and $E_{W_{g}}$ is the weight error for the $g$ th group of weights and biases, and $G$ is the number of groups of weights and biases in the neural network.

\subsection{Bayesian interference}

The adaptive parameters of neural networks (weights and biases) can be co nveniently grouped into a single $W$-dimensional weight vector $w$. According to the Bayesian 


\section{Hung Tan Nguyen}

inference, the posterior distribution of the weight vector $w$ of a neural network given a data set $D$ is given by

$$
p(w \mid D, \psi)=\frac{p(D \mid w, \psi) p(w \mid \psi)}{p(D \mid \psi)}
$$

where $\psi=\left\{\xi_{1}, \ldots, \xi_{G}\right\}$ and equation (3) is the first level of the inference. $p(w \mid \psi)$ is the weight prior determined using the theory of prior.

Using the Ba yes' theorem, we can e xpress the posterior distribution of the hyperparameters as

$$
p(\psi \mid D)=\frac{p(D \mid \psi) p(\psi)}{p(D)} \equiv p(D \mid \psi) p(\psi)
$$

where $p(\psi)$ is the prior distribution of the hyperparameters and simply we assume that this distribution is uniform.

Rearranging (3), we have the following form:

$$
p(D \mid \psi)=\frac{p(D \mid w, \psi) p(w \mid \psi)}{p(w \mid D, \psi)}
$$

Taking the derivative of $\ln p(D \mid \psi)$ with respect to $\xi_{g}$

$$
\frac{\partial}{\partial \xi_{g}} \ln p(D \mid \psi)=\frac{W_{g}}{2 \xi_{g}}-E_{W_{g}}-\frac{1}{2} \operatorname{tr}\left(A^{-1}\right) I_{g}
$$

Let this derivative be zero, we can determine $\xi_{g}$ as follows

$$
2 \xi_{g} E_{W_{g}}=W_{g}-\xi_{g} \operatorname{tr}\left(A^{-1}\right) I_{g}
$$

The right-hand side is equal to a value $\gamma_{g}$ defined as

$$
\gamma_{g}=W_{g}-\xi_{g} \operatorname{tr}\left(A^{-1}\right) I_{g}
$$

$\gamma_{g}$ is called the number of well-determined parameters in weight group $g$. Substituting (8) into (7) and rearranging (7), we have

$$
\xi_{g}=\frac{\gamma_{g}}{2 E_{W_{g}}}
$$

The terms $\xi_{g}$ and $\gamma_{g}$ are used with some formulas to compute the logarithm of the evidence in Bayesian model comparison. The optimal model is selected corresponding to the highest logarithm of the evidence [Thodberg 1996; Bishop 1995].

\subsection{Parameter optimisation algorithms for Bayesian neural networks}

The main problem when training neural networks is that usually suitable values for the learning rate and momentum must be chosen. As this procedure is clearly inefficient, we focus on fast training algorithms which can automatically determine the search direction and step size. Three advanced training algorithms for Bayesian neural network classifiers 
Intelligent Technologies for Real-time Biomedical Engineering Applications

are used for the training of algorithms. They are conjugate gradient, quasi-Newton and scaled conjugate gradient algorithms [Bishop, 1995].

\section{Results}

\subsection{Non-invasive hypoglycaemia monitor}

Twenty-five children with T1DM (14.4 \pm 1.6 years) volunteered for the 4-hour glucose clamp study to provide 28 sets of physiological responses at the Princess Margaret Hospital for Ch ildren in Perth, Australia. Data were collected with ap proval from Women's and Children's Health Service, Department of Health, Government of Western Australia, and with informed consent.

Each study consists of five phases: baseline (30 min), euglycemia (60 $\mathrm{m}$ in), ramp phase (30 $\mathrm{min})$, hypoglycemia (40 $\mathrm{min})$ and euglycemia $(30 \mathrm{~min})$ as shown in Figure 1. HypoMon was used to measure the required physiological parameters, while the actual blood glucose (BG) levels were collected as reference using Yellow Spring Instruments. The main parameters used for the detection of hypoglycemia are the heart rate, corrected QT interval and skin impedance.

The HypoMon (Hypoglycemia Monitor) as shown in Fig. 2 was used to measure the relevant physiological responses (heart rate, corrected QT interval of the ECG signal, skin impedance), while the actual blood glucose (BG) measurements were collected a s reference. The four skin-surface bio-sensor electrodes are multiplexed and shared to measure both skin impedance and ECG signals.

Figure 2 HypoMon

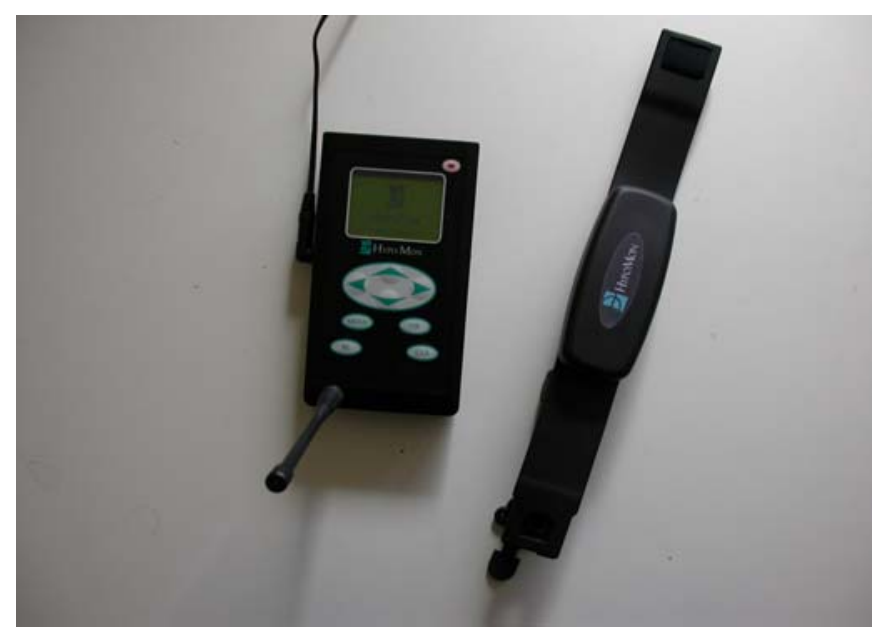


Hung Tan Nguyen

The responses from $25 \mathrm{~T} 1 \mathrm{DM}$ children exhibit significant changes during the hypoglycemia phase against the non-hypoglycemia phase. Normalisation was used to reduce patient-to-patient variability and to enable group comparison by dividing the patient's heart rate, corrected QT interval and skin impedance by his/her corresponding values at time zero. The study shows that associated with hypoglycemic episodes in 25 T1DM children, using normalized values, their heart rates increas e significantly $(1.152 \pm 0.157$ vs. $1.035 \pm 0.108, \mathrm{P}<0.0001)$, their corrected QT in tervals increase significantly $(1.088 \pm 0.086$ vs. $1.020 \pm 0.062, \mathrm{P}<0.0001)$, and their skin impedances reduce significantly $(0.679 \pm 0.195$ vs. $0.837 \pm 0.203, \mathrm{P}<0.0001)$.

The detection of hypoglycemic episodes ( $\mathrm{BG}<=60 \mathrm{mg} / \mathrm{dl}$ or $3.33 \mathrm{mmol} / \mathrm{l}$ ) using these three variables is based on an optimal Bayesian neural network algorithm developed from the obtained clinical data. This neural network has a multilayer feed-forward neural network structure with one input layer, one hidden layer and one output layer. In effect, it estimates the presence of hypoglycemia at sampling period $\mathrm{k}$ based on the basis of the data at sampling period $\mathrm{k}$ and the previous data at sampling period $\mathrm{k}-1$. In general, the sampling period is 5 minutes and approximately 30 data points are used for each patient.

The overall data set consisted of a traini ng set a nd a test set, each with 14 cases randomly selected. For these, the whole data set which included both hypoglycemia data part and non-hypoglycemia data part were used. For optimal robustness of the evaluation, we applied the evidence framework for Bay esian inference to the training set and found the feed forward neural network architecture with 11 hidden nodes yielded the highest evidence. We found among the three advanced training algorithms as discussed earlier, Quasi-Newton technique was the most effective for this application. The optimisation of Bayesian neural network architecture is important for on-line network training systems because it can contribute to the least network training time while still maintaining the best generalisation for the network. The final feed-forward multi-layer neural network had heart rate, corrected QT interval and skin impedance as inputs, 11 hidden nodes and 1 output node (estimated blood glucose level). From this optimal neural network which was derived from the training set, the estimated BG profiles produced a significant correlation $(\mathrm{P}<0.0001)$ against measured values.

The corresponding ROC Curve area for the training set was 0.9135 wih $95 \%$ CI of $(0.8748,0.9521)$. For an equal value of sensitivity and specificity of the training set, the optimal cut-off point selected in this study was chosen to be -0.09082 . The selected neural network algorithm was then applied to the test set $(14$ cases). It produced a sensitivity of 0.8346 (true positive) and a specificity (true negative) of 0.6388 .

\subsection{Power wheelchairs}

Data were collected from eight adults, age d between 19 and 56, with approval from the UTS Human Research Ethics Committee and informed consent from the volunteers. Of these, four had high-level spinal cord injuries (C4 and C5) and were not able to use a standard joystick to control a wheelchair. The remaining four did not have conditions affecting their head movement. Data for each person was collected in two periods of ten minutes, with the user being prompted to give a specified movement every 6 seconds. 
Intelligent Technologies for Real-time Biomedical Engineering Applications

Each specified movement was chosen randomly from the following: forward, backward, left and right. The extracted movement samples of those users are shown in Table 1.

Table 1 Extracted movement samples

\begin{tabular}{|l|l|l|l|l|l|}
\hline User & Forward & Backward & Left & Right & $\begin{array}{l}\text { Injury } \\
\text { Level }\end{array}$ \\
\hline 1 & 20 & 20 & 20 & 20 & - \\
\hline 2 & 20 & 20 & 20 & 20 & - \\
\hline 3 & 20 & 20 & 20 & 20 & - \\
\hline 4 & 20 & 20 & 20 & 20 & - \\
\hline 5 & 20 & 20 & 20 & 20 & $C 5$ \\
\hline 6 & 20 & 20 & 20 & 20 & $C 4$ \\
\hline 7 & 20 & 20 & 20 & 20 & $C 4$ \\
\hline 8 & 20 & 20 & 20 & 20 & $C 5$ \\
\hline
\end{tabular}

Different Bayesian neural networks with varying numbers of hidden nodes were trained to select the optimal network architecture. These networks have the following specification:

- four hyperparameters $\xi_{1}, \xi_{2}, \xi_{3}$ and $\xi_{4}$ to constrain the magnitudes of the weights;

- 41 inputs, corresponding to 20 samples from $x$ axis, 20 samples from $y$ axis and one augmented input with a constant value of 1 ;

- four outputs, each corresponding to on e of the classes: forward, backward, left and right movement.

Training data were taken from the recorded movements of Users 1, 2, 3, 4, 5 and 6, corresponding to 480 sample patterns (Table 1). For a given number of hidden nodes, ten networks with different initial conditions were trained using the quasi-Newton training algorithm. As shown in Fig. 3, the networks with three hidden nodes have the highest evidence. These networks also have low test errors (misclassification percentage). 
Hung Tan Nguyen

Figure 3 Log evidence versus number of hidden nodes

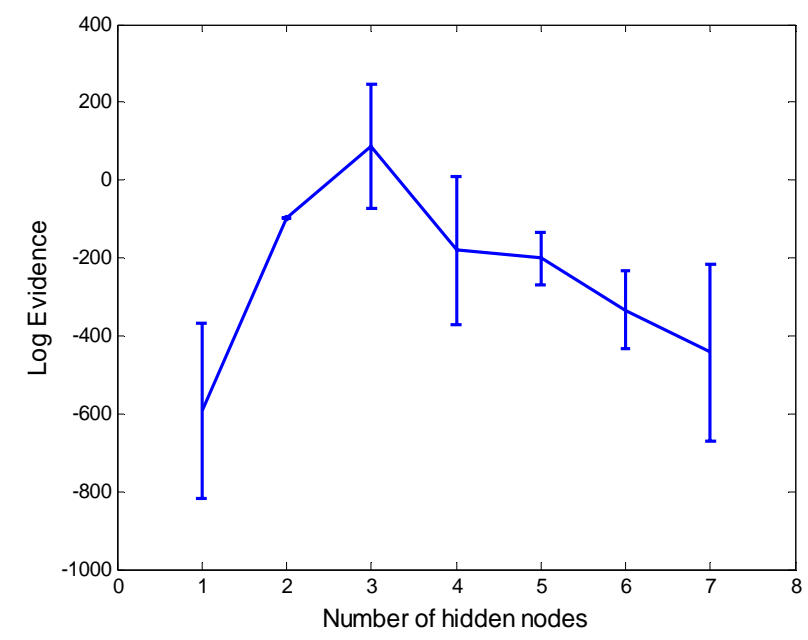

Again, the optimisation of Bayesian neural network architecture is extrem ely important for on-line network training systems because in corporating with the Quas iNewton training algorithm, it can contribute to the least network training time while still maintaining the best generalisation for the network. This combined property would be ideal for on-line and real-time implementation.

\section{Experiment $I$}

The training data were taken from the recorded movements of Users 1, 2, 3, 4, 5 and 6 (480 sample patterns total). The recorded movements of Users 7 and 8 were ra ndomly divided into two sets. Eac $\mathrm{h}$ set containe d 20 sam ple patterns of each $\mathrm{m}$ ovement. A Bayesian neural network classifier having the optimal number of three hidden nodes was trained using the above training data.

The performance of the trained network was tested using the first set of movement samples of Users 7 and 8 ( 80 sample patterns total). The confusion matrix in Table 4.2 shows how that the trained network can classify all sa mples in the test set with a sensitivity (true positive) of $85 \%$ and a specificity (true negative) of $95 \%$. Note that head movement samples of Users 7 and 8 were not included in the training data.

\section{Experiment II}

This Experiment is an extension of the above. More training data was taken from the second set of movement samples of Use rs 7 a nd 8 (80 sample patterns total). The performance of the trained network was again tested using the first set of movement samples of Users 7 and 8. Similarly, a confusion matrix used to evaluate the performance 
Intelligent Technologies for Real-time Biomedical Engineering Applications

of the trained network is shown in Table 2. This time, the revised network can classify all samples in the test set with a sensitivity of $93.75 \%$ and a specificity of $97.92 \%$.

The classification results of Experiment II are summarised in Table 2. Especially, it can be seen that very high sensitivity and specificity have been achieved. This means that the performance of the trained network has been significantly improved as $\mathrm{m}$ ore movement samples have been included to train the network.

Table 2 Confusion matrix in Experiment II

\begin{tabular}{llllll}
\hline \hline & \multicolumn{5}{l}{ Predicted Classification } \\
\hline & Movement & Forward & Backward Left & Right \\
\hline \multirow{3}{*}{ Actual } & Forward & 18 & 0 & 1 & 1 \\
Classification & Backward & 0 & 19 & 1 & 0 \\
& Left & 2 & 0 & 18 & 0 \\
& Right & 0 & 0 & 0 & 20 \\
\hline \hline
\end{tabular}

Figure 4 Hands-free control of power wheelchair

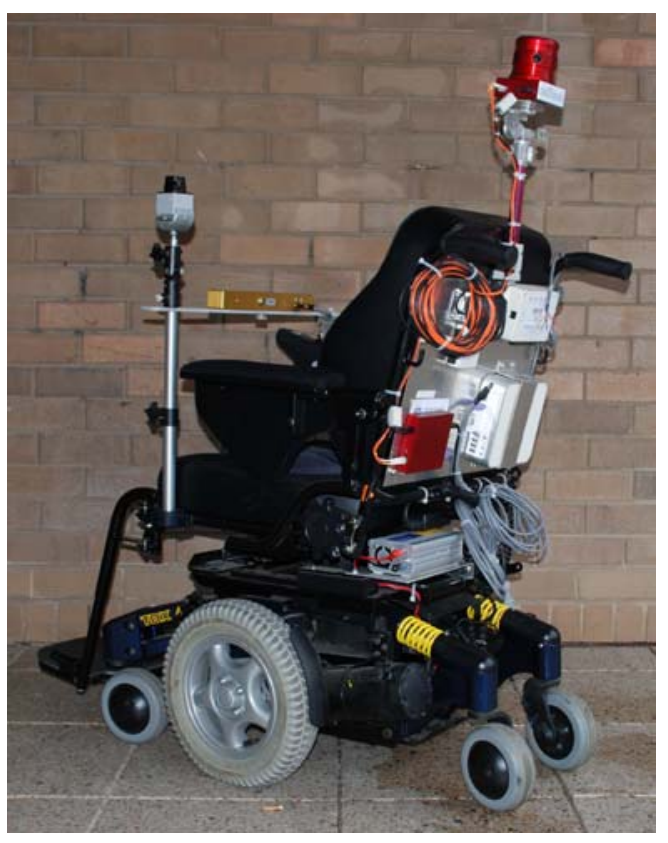


H ung Tan Nguyen

\section{Conclusion}

The above result indicates that hypoglycemic episodes in T1DM children can be detected non-invasively and continuously effectively from the real-time physiological responses measured by HypoMon. In th is study, the sen sitivity obtained by the hypoglycemia detection neural network is good but its overall accuracy could be improved. A m ore advanced neural network algorithm will be developed to improve its accuracy in the near future.

The results obtained also show that Bayesian neural networks can be used to classify head movement accurately. The use of three hidden nodes is an op timal choice for the network architecture and the available training data.

When the Bayesian neural network was trained using head movement data of the six users (four able-bodied, two disabled persons), it can classify head movements of two new disabled persons with $85 \%$ sensitivity and $95 \%$ specificity. However, if the network was trained further with additional head movement samples of those two new disabled persons, it can classify th eir head movements with a high sensitivity of $93.75 \%$ while retaining an excellent specificity of $97.92 \%$. In other words, the optimal Bayesian neural network was able to provide an on-line adaptation to the head direction movements of new disabled wheelchair users effectively.

\section{Acknowledgement}

I thank Dr Nejhdeh Ghevondian, Assoc Prof Timothy Jones, Dr Son Nguyen, Dr Phil Taylor and Assoc Prof James Middleton for their contributions.

\section{References}

Bishop, M., (1995) Neural networks for pattern recognition, Oxford: Clarendon Press, New York. Clarke, W.L., Cox, D.J., Gonder -Frederick, L.A,, Julian, D., Schlundt, D., Polon sky. W. (1995), 'Reduced awareness of h ypoglycemia in adults with IDDM. A prospectiv e study of hypoglycemic frequency and associated symptoms', Diabetes Care, 18, pp. 517-522.

Coyle, E.D. (1995), 'Electronic wheelchair controller designed for operating by hand-operated joystick, ultrasonic non-contact head contro 1 and utteran ce from a small wo rd-command vocabulary,' IEE Colloqium (Digest), 55: 3/1-3/4.

Craig, D.A., Ng uyen, H.T. Nguyen (2005), 'Wireless real- time head movement s ystem using a Personal Assistant (PDA) for control of a po wer wheelchair', 27 $7^{\text {th }}$ Annual International Conference of the IEEE Engineering in Medicine and Biology Society, Shanghai, China, 1-4 September, paper 814.

Craig, D.A., Nguyen, H.T., Burchey, H.A. (2006), 'Control of Power Wheelchair using EEG-based Thought Pattern Recognition', $28^{\text {th }}$ Annual International Conference of the IEEE Engineering in Medicine and Biology Society, New York City, USA, A ugust 31-September 3, pp. 12911294.

Craig, D.A., N guyen, H.T. (2007), 'Adaptive EEG Thought Pattern Cla ssifier for Advanced Wheelchair Control', $29^{\text {th }}$ Annual International Conference of the IEEE Engineering in Medicine and Biology Society, Lyon, France, August 23-26, pp. 2544-2547.

Cryer, P.E. (2002), 'Hypoglycaemia: the limiting factor in the glycaemic management of type I and type II diabetes', Diabetologia, Vol. 45, pp. 937-948. 


\section{Intelligent Technologies for Real-time Biomedical Engineering Applications}

Davis, S., Alonso, M.D. (2004), 'Hypoglycemia as a barrier to glycemic control'. J. of Diabetes and its Complications, Vol. 18, pp. 60-68.

DCCT Research Group (1993) 'The eff ect of intensive treatment of diabetes on the development and progression of long-term complications in IDDM'. N. Eng. J. Med., 329, pp. 977-986.

DCCT Research Group (1995) 'Adverse events and their association with treatment regimens in the Diabetes Control and Complications Trial', Diabetes Care, 18, pp. 1415-1427.

DCCT Research Group (1991), 'Epidemiology of severe hypoglycemia in the diabetes control and complication trial', Am. J. Med., 90, pp. 450-459.

Gale E.A.M., Bennett, T., MacDonald, I.A., Holst J.J. and Matthews, J.A. (1983), 'Th e physiological effects of insulin-induced hypoglycemia in man: responses at differing levels of blood glucose', Clin. Sciences, 65, pp. 263-271.

Ha, Q.P., Tran, T.H., Dissanay ake, G. (2005), 'A wavelet- and neural network-based voice interface system for wheelchair contro 1', Int. J. Intellig ent Systems Technologies an $d$ Applications, Vol. 1, Nos. 1/2, pp. 49-65.

Heller, S.R. and Macdonald, I.A. (1991), 'Physiological disturbances in hypoglycemia: effect on subjective awareness', Clin. Sci., 81, pp. 1-9.

Harris, N.D., Bay kouchev, S.B., Marques, J.L, (1996), 'A p ortable system for monitoring physiological responses to hypoglycemia', J. Med.Eng. \& Tech., Vol. 20, pp. 196-202.

Joseph, T. and Nguyen, H. T. (1998), 'Neural network control of wheelchairs using telemetric head movement', Proceedings of the 20th Annual International Conference of the IEEE, Engineering in Medicine and Biology Society, Vol. 5, pp. 2731 - 2733.

Marques, J.L., George, E., et al. (1997), Altered ventricular repolarisation during hypoglycaemic in patient with diabetes, Diabetic Med; 8, pp. 648-654.

MacKay, D. (1 992a), The evidence framework applied to classification networks. Neural Computation, 4, pp. 720-736.

MacKay, D. (1992b). A practical Bay esian framework for back-propagation networks, Comp. \& Neural Systems, 4, pp. 448-472.

Mazo, M., Rodrigues, F..J., Lazoro, J.L., Urena, J., Garcia, J.C., Santino, E., Revenga, R.A.. (1995), 'Electronic control of a wheelchair g uided by voice commands', Control Eng. Practice, 3, pp. 665-674.

Nguyen, H.T., King, L.M. and Knight. G. ( 2004), 'Real-time head movement s ystem and embedded Linux implementation for the co ntrol of power wheelchairs,' 26th Annual International Conference of the Engineering in Medicine and Biology Society, Vol. 2, pp . 4892 - 4895.

Nguyen H.T., Ghevondian, N., Jones, T. (200 6a), 'Neural-network detection of hypoglycemic episodes in children with type 1 diabetes using physiological parameters', $28^{\text {th }}$ Annual Int Conf of the IEEE Eng in Med and Biol Society, New York, pp. 6053-6056.

Nguyen, S.T., Nguyen, H.T., Taylor, P.B., Middleton, J. (20 06b), 'Improved Head Direction Command Classification using an Optim ised Bayesian Neural Network', $28^{\text {th }}$ Annual International Conference of the IEEE Engineering in Medicine and Biology Society, New York City, USA, August 31-September 3, pp. 5679-5682.

Nguyen, H.T., Nguyen, S.T., Taylor P.B., Middleton, J. (2007), 'Head Direction Command Classification using an Adaptive Optimal Bayesian Neural Network', International Journal of Factory Automation, Robotics and Soft Computing, Issue 3, July 2007, pp. 98-103.

Penny, W.D., Roberts, S.J. (1999), 'Bayesian neural networks for classification: how useful is the evidence framework', Neural Networks, 12, pp.877-892.

Simpson, R.C., Levine, S.P. (2002), 'Voice control of a powered wheelchair', IEEE Trans. Neural Systems and Rehabilitation Engineering, June, Vol. 10, No. 2, pp. 122-125.

Thodberg, H.H, (1996), A review of Bayesian neural networks with an application to near infrared spectroscopy, IEEE Transactions on Neural Networks, Vol. 7, pp. 56-72.

Yale J-F (2004) 'Nocturnal hypoglycemia in patients with insulin-treated diabetes', Diab Research and Clinical Practice, 65S:S41-S46. 